\title{
Autoestima y dependencia emocional en relaciones de pareja de estudiantes universitarios
}

\section{Self-esteem and emotional dependence in partner relationships in university students}

\author{
Itzel X. Quiroz-Fonseca ${ }^{a}$, Norma A. Ortega-Andrade ${ }^{b}$,Merali G. Godínez-Gutiérrez ${ }^{c}$, Angélica \\ M. Jahuey-Riaño ${ }^{d}$, María L. Montes-Hernández ${ }^{e}$
}

\begin{abstract}
:
Some studies have shown that low self-esteem can be one of the causes associated with emotional dependence, others have reported a low relationship between these two psychological conditions. Therefore, the present research aimed to identify the level of selfesteem and emotional dependence in university students and in this way correlate both variables, seeing if one affected the other. Eighty-two undergraduate psychology students from a public university participated, who were at the time of the study, in a relationship with a partner lasting at least 4 months. These were selected through a non-probability convenience sampling. The research design was non-experimental, cross-sectional, with correlational scope. The Rosenberg Self-esteem Scale and the Relationship Behavior Scale validated for the Mexican population were used. The results report a low level of self-esteem $M=24$ $(S D=5.06)$ and an average level of emotional dependence at the 19 th percentile, with $81 \%$ of them being above this value, indicating the presence of emotional dependence. Regarding the relationship between self-esteem and emotional dependence, mean and inversely proportional correlations of self-esteem were found with the following traits of emotional dependence: desire for exclusivity in the relationship $\left(r_{s}=-0.380, \mathrm{p} \leq 0.01\right)$, relationships based on submission and subordination $\left(r_{s}=-0.223, \mathrm{p} \leq 0.01\right)$, history of unbalanced relationships $\left(r_{s}=-0.448, \mathrm{p} \leq 0.01\right)$, fear of breakup $\left(r_{s}=-0.350, \mathrm{p} \leq 0.01\right)$, characteristics of the objects of emotional dependents $\left(r_{s}=\right.$ $-0.398, \mathrm{p} \leq 0.01)$ and dominant dependence $\left(r_{s}=-0.364, \mathrm{p} \leq 0.01\right)$ and a low and inversely proportional correlation of self-esteem with the emotional dependence trait, idealization of the object $\left(r_{s}=-0.224, \mathrm{p} \leq 0.05\right)$, that is, the lower the self-esteem, the greater the emotional dependence. There was no correlation between self-esteem and excessive need for the other $\left(r_{s}=-0.210, \mathrm{p}=0.058\right)$ and self-esteem with the dimension of prioritizing the partner over anything else $\left(r_{s}=-0.164, \mathrm{p}=0.139\right)$. These findings indicate that there is a relationship between self-esteem and emotional dependence, which is why the research hypothesis is accepted. It is concluded to observe the results with reserve, considering that the psychometric properties of the instruments need to be further studied
\end{abstract}

\section{Keywords:}

Self-esteem, emotional dependence, partner relationship

Resumen:

Algunos estudios han evidenciado que la baja autoestima puede ser una de las causas asociadas a la dependencia emocional, otros han reportado una baja relación entre estas dos condiciones psicológicas. Por lo cual, la presente investigación tuvo como objetivo identificar el nivel de autoestima y dependencia emocional en estudiantes universitarios y de esta manera correlacionar ambas variables viendo si alguna afectaba a la otra. Participaron 82 estudiantes de la licenciatura en psicología de una universidad pública, que se encontraban al momento del estudio, en una relación de pareja con al menos 4 meses de duración. Éstos fueron seleccionados a través de un muestreo no probabilístico por conveniencia. El diseño de investigación fue no experimental transversal, con alcance

\footnotetext{
Autor de Correspondencia, Universidad Autónoma del Estado de Hidalgo, https://orcid.org/0000-0002-6343-7926, Email: qu356871@uaeh.edu.mx

b Universidad Autónoma del Estado de Hidalgo, https://orcid.org/0000-0002-0117-2547, Email: ortegan@uaeh.edu.mx

c Universidad Autónoma del Estado de Hidalgo, https://orcid.org/0000-0002-6605-8649, Email: go359919@uaeh.edu.mx

d Universidad Autónoma del Estado de Hidalgo, https://orcid.org/0000-0003-3759-4810, Email: ja299337@uaeh.edu.mx

e Universidad Autónoma del Estado de Hidalgo, https://orcid.org/0000-0001-9951-9504, Email: mo359920@uaeh.edu.mx
} 
correlacional. Se utilizaron la Escala de Autoestima de Rosenberg y la Escala de conducta en la relación de pareja validados para población Mexicana. Los resultados reportan un nivel de autoestima bajo $M=24(D E=5.06)$ y un nivel de dependencia emocional promedio en el percentil 19, siendo un $81 \%$ quienes se encuentran por arriba de este valor, indicando la presencia de dependencia emocional. Respecto de la relación entre autoestima y dependencia emocional, se encontró correlaciones medias e inversamente proporcionales de la autoestima con los siguientes rasgos de dependencia emocional: deseo de exclusividad en la relación $\left(r_{s}=-0.380\right.$, $\mathrm{p} \leq 0.01)$, relaciones basadas en la sumisión y subordinación $\left(r_{s}=-0.223, \mathrm{p} \leq 0.01\right)$, historia de relaciones de pareja desequilibradas $\left(r_{s}\right.$ $=-0.448, \mathrm{p} \leq 0.01)$, miedo a la ruptura $\left(r_{s}=-0.350, \mathrm{p} \leq 0.01\right)$, características de los objetos de los dependientes emocionales $\left(r_{s}=-0.398\right.$, $\mathrm{p} \leq 0.01)$ y dependencia dominante $\left(r_{s}=-0.364, \mathrm{p} \leq 0.01\right)$ y una correlación baja e inversamente proporcional de autoestima con el rasgo de dependencia emocional, idealización del objeto $\left(r_{s}=-0.224, \mathrm{p} \leq 0.05\right)$, esto es, a menor autoestima mayor dependencia emocional. No hubo correlación entre autoestima y necesidad excesiva del otro $\left(r_{s}=-0.210, \mathrm{p}=0.058\right)$ y autoestima con la dimensión de la priorización de la pareja sobre cualquier otra cosa $\left(r_{s}=-0.164, \mathrm{p}=0.139\right)$. Estos hallazgos indican que existe una relación entre autoestima y dependencia emocional, por lo que se acepta la hipótesis de investigación. Se concluye observar los resultados con reserva, al considerar que las propiedades psicométricas de los instrumentos necesitan seguirse estudiando.

\section{Palabras Clave:}

Autoestima, dependencia emocional, relación de pareja

\section{Introducción}

En las relaciones de pareja el amor es una expresión de sentimientos que se manifiesta de diferentes formas; algunas, tienden a conducirse en relaciones amorosas basadas en la reciprocidad, en la comunicación, la confianza y el respeto mutuo, otorgando satisfacción, bienestar psicológico y social en cada encuentro que sostiene con el otro; otras en cambio, se fundamentan en los celos excesivos, el maltrato físico y verbal, el vacío emocional, o bien, la dependencia emocional.

En el caso específico de las relaciones, donde una de las partes establece un vínculo amoroso basado en la dependencia emocional; el temor constante a perder a la pareja y la demanda excesiva y exclusiva de la otra persona, provocan que la relación se torne en una serie de demandas afectivas insatisfechas que se intentan cubrir de manera desadaptativa con la pareja $(1,2,3)$, llegando incluso al sometimiento y la subordinación, soportando desprecios y humillaciones por miedo a perder a la pareja objeto de su amor (4).

Regularmente las personas que son dependientes emocionalmente buscan parejas que son egocéntricas, seguras de sí mismas, dominantes y poco afectuosas, como una conducta compensatoria a su baja autoestima y temor. En palabras de Castello (5), buscan parejas con esas características, porque las idealizan a tal grado que llegan a verles como dioses o seres excepcionales, sin percatarse que detrás de esa persona encumbrada a extremos inimaginables, muchas veces no hacen otra cosa que hacerle la vida difícil a la persona que es dependiente emocionalmente.

De acuerdo con Moral y Sirvent (6) la dependencia emocional puede definirse como "un patrón crónico de demandas afectivas frustradas, que buscan desesperadamente satisfacerse mediante relaciones interpersonales de apego patológico" (6).
Castelló en 2005 hizo referencia a la dependencia emocional como un trastorno de la personalidad basado en el Manual Diagnóstico de los Trastornos Mentales (DSM-IV) (7) dado que según él, cumplía con ciertos criterios atribuibles a este tipo de trastorno, sin embargo en su momento, también marco una diferencia con éste en que la persona dependiente emocionalmente, muestra una "una tendencia persistente a las relaciones de pareja caracterizadas por el desequilibrio entre ambos miembros, la necesidad afectiva claramente excesiva hacia la otra persona y el sometimiento inapropiado hacia ella, que empieza al principio de la edad adulta y se da en diversos contextos". (5), por lo que la ubicó en una categoría a la que denominó Trastorno de la Personalidad por Necesidades Emocionales. No obstante, en el DSM-V (8), no se contempla dicha categoría, por lo que no se podría confirmar esta propuesta.

Por consiguiente, acorde con Castelló (5), las características de las personas dependientes emocionalmente son: necesidad excesiva del otro; deseo constante hacia la pareja; deseos de exclusividad en la relación; prioridad de la pareja sobre cualquier otra cosa; idealización del objeto, siendo la pareja todo aquello que el dependiente no tiene (seguridad en sí mismo, autoaprecio y una posición de superioridad sobre los demás). Mantienen un concepto distorsionado de lo que significa el amor y de lo que supone una relación de pareja, teniendo como consecuencia, historias de relaciones de pareja desequilibradas provocando en la persona que la padece perturbación y sufrimiento en torno al tema del amor que mantienen específicamente con la pareja, presentando efectos negativos hacia la percepción de sí mismos y su valía como personas.

Rodríguez-Franco, Rodríguez-Díaz, Antuña Bellerín y López-Cepero (9), consideran que el problema de la dependencia emocional surge en la etapa en la que 
se inician las relaciones de pareja (como lo es la adolescencia o la juventud tardía), como resultado de la creencia de una entrega incondicional al otro y la aceptación de aspectos placenteros y doloroso como parte de la relación misma (10).

Estudios en torno a la dependencia emocional en estudiantes universitarios y su relación con factores psicológicos como la autoestima, reportan una asociación negativa entre éstas, donde a mayor dependencia emocional menor nivel de autoestima (11).

Además con respecto a las diferencias en cuanto al sexo, el estudios realizado por Urbiola, et al. (11), no mostró diferencias en cuanto a la dependencia total; sin embargo, los hombres presentaron puntuaciones más altas en la necesidad de agradar y obtener la aprobación de los demás. También encontraron puntuaciones altas en la necesidad de exclusividad y evitar estar solo.

Otra investigación respecto al género evidencia que los varones suelen expresar su dependencia emocional de forma agresiva hacia su pareja, en tanto las mujeres, recurren con mayor frecuencia al autoengaño y el uso de mecanismos de negación y no afrontamiento (12).

Un estudio realizado por Marín-Ocmin (13), con respecto a la relación entre dependencia emocional y autoestima encontró que los estudiantes que presentan una autoestima alta no reportan dependencia emocional. También, que la dependencia emocional estaba asociada al sexo, la edad y el tipo de familia; por lo que afirma que las personas con dependencia emocional no tienen afecto hacia ellas mismas, se sienten indefensas, necesitadas y vulnerables en cuanto al área afectiva. Respecto del sexo, este mismo estudio evidenció que las mujeres mostraron menor dependencia emocional que los hombres.

En relación a la dependencia emocional y sus repercusiones en la autoestima, Llanes y Leal (14) mencionan que ésta puede variar acorde con la seguridad que le ofrezca la figura que le genera dependencia, esto es, que la autoestima de una persona puede variar en función de la conexión que exista con su pareja inmediata. De acuerdo con Hertfelder (15), la baja autoestima y la inseguridad que se observa en las personas con dependencia emocional, pueden ser consecuencia de experiencias tempranas en el núcleo familiar donde ha faltado un apego seguro, por lo que buscan obtenerlo en su pareja.

En este sentido Riso en 2003, ya había escrito que las personas con dependencia afectiva buscan suplir los vacíos afectivos producto de las relaciones de apego inseguro con las figuras primarias, manteniendo vínculos insanos con la pareja, al crear una necesidad por el otro y el deseo de estar en compañía con él (16).

Con respecto a los efectos de la Dependencia Emocional que se pueden observar en los estudiantes universitarios, son: bajo rendimiento académico, desgano, desmotivación, depresión, sentimientos de inferioridad, aislamiento $(17,18)$, y cuando no tienen una orientación adecuada o la ayuda necesaria pueden a atentar contra su propia integridad física.

Se considera que la autoestima, como un sentimiento valorativo del nuestro ser, de quiénes somos nosotros, del conjunto de rasgos corporales, mentales y espirituales que configuran nuestra personalidad (2) debe atenderse en los ámbitos educativos de tal manera que no se tenga una visión negativa de sí mismo, propiciando inseguridad y por ende dependencia emocional en algunos jóvenes.

Es preciso saber que la dependencia emocional, afecta considerablemente a la persona que lo vive y a la persona que se encuentra a su lado, debido a la presencia de celos patológicos, dominio y control sobre la pareja; disminución de la autoestima, depresión, ansiedad y trastornos alimentarios como anorexia y bulimia en algunos casos, o bien, el consumo de sustancias adictivas en otros.

Por ello, la necesidad de indagar la presencia de este tipo de conductas en las relaciones de parejas de los estudiantes universitarios, quienes en algún momento, podrían verse afectados por este tipo de comportamientos y presentar repercusiones en su proceso de aprendizaje. De igual forma, los resultado de estudios en donde se ha encontrado una relación significativa entre la autoestima y la dependencia emocional y otros estudios donde la relación entre éstas variables no es significativa (19), muestran resultados contradictorios que llevan a plantear la necesidad de seguir investigando sobre este tema.

Por lo cual, el presente estudio tuvo como objetivo evaluar la asociación que existía entre la autoestima y la dependencia emocional en los estudiantes universitarios de psicología de una universidad pública que sostenían una relación de pareja, a fin de aportar información que en un momento inmediato, pudiera ser útil en la atención primaria de la salud emocional.

Como objetivos específicos, se estableció identificar en qué nivel de autoestima se encontraban los estudiantes, así como el nivel de dependencia emocional que presentaban en su relación de pareja, como el tipo de rasgo de dependencia emocional.

En el estudio se planteó la hipótesis de una relación estadísticamente significativa entre la autoestima y la dependencia emocional en la relación de las parejas de estos estudiantes universitarios.

\section{Metodología}

\section{Participantes}


En el estudio participaron 82 estudiantes de la licenciatura de psicología de un universidad pública quienes se encontraban cursando de $3^{\circ}$ a $9^{\circ}$ semestre, y al momento del estudio mantenían una relación de noviazgo mayor a 4 meses. Los participantes fueron seleccionados mediante un muestreo no probabilístico por conveniencia, una vez que aceptaron formar parte del estudio con la firma del asentimiento informado. El diseño de la investigación fue no experimental, transversal, con alcance correlacional.

\section{Variables e Instrumentos}

La autoestima se midió con la Escala de Autoestima de Rosenberg del autor Morris Rosenberg publicada en 1965. Consta de 8 ítems en la validación para población mexicana, cada uno de ellos es una afirmación sobre la valía personal y la satisfacción con uno mismo. Tres frases están enunciadas de forma positiva y cinco de forma negativa.

Los ítems 2, 4 y 5 , las respuestas $A$ a $D$ se puntúan de 4 a 1 . De los ítems del 6 al 10, las respuestas $A$ a $D$ se puntúan de 1 a 4 . La escala fue traducida y validada en castellano por Jurado D., Jurado C., López, Querevalú (20). La consistencia interna de la escala se encuentra entre el 0.76 y 0.87 . La fiabilidad es de 0.80

De acuerdo con las normas de calificación reportadas por estos autores, de 31 a 32 puntos significa que la persona tiene un nivel de autoestima muy alto. De 29 a 30 un nivel de autoestima alto; de 26 a 28 puntos significa que tiene una autoestima leve y no presenta problemas de autoestima grave, pero es conveniente mejorarla. Un puntaje de 1 a 25 puntos representa una autoestima baja, lo cual se considera que existen problemas significativos de autoestima.

La dependencia emocional fue medida con la Escala de conducta en la relación de pareja diseñado y validado por Arellano en 2010 para población mexicana con un rango de edad de 20 a 30 años. La confiabilidad del instrumento es igual a un alfa de $0.91 \mathrm{La}$ validez de los reactivos se encuentra entre 0.30 y 0.85 con un promedio general de 0.54 para todo el instrumento.

Este instrumento consta de 59 reactivos, distribuidos de acuerdo a los rasgos de dependencia emocional que mide:

- Necesidad excesiva del otro, deseo constante de acceso a él /ella.

- Deseo de exclusividad en la relación.

- Prioridad de la pareja sobre cualquier otra cosa

- Idealización del objeto.

- Relaciones basadas en la sumisión y subordinación.

- Historia de relaciones de pareja desequilibradas.

- Miedo a la ruptura.
- Características de los objetos de los dependientes emocionales.

- Dependencia dominante.

La escala tiene 4 respuestas Totalmente de acuerdo (TA), De acuerdo (A), En desacuerdo (D) y Totalmente en desacuerdo (TD), las cuales TA y A son respuestas afirmativas y se puntúan con 1 y $D$ y TD son respuestas negativas y se puntúan con 0 , posteriormente se suman los puntos para obtener un puntaje total que indica el nivel de dependencia emocional que muestra persona.

\section{Procedimiento}

Se solicitó la autorización de a la autoridad de la institución, para poder aplicar las escalas a los alumnos de $3^{\circ}$ a $9^{\circ}$ semestre. Una vez que se contó con su aprobación, se acudió a las aulas y se informó a los profesores sobre el estudio con la finalidad de poder aplicar las escalas frente al grupo. Ya presente con los alumnos, se les explicó brevemente los objetivos de la investigación y se les invitó a participar en la misma, una vez que se identificó a quienes tenían una relación con una duración mayor a 4 meses. Posteriormente se les proporcionaron las instrucciones para contestar las escalas, además del consentimiento informado. Una vez que concluyeron se agradeció su participación.

Después de la aplicación de escalas, se procedió a realizar los análisis estadísticos en el Paquete Estadísticos para las Ciencias Sociales (SPSS) para analizar los resultados.

\section{Metodología}

Con la finalidad de identificar el nivel de autoestima y el rasgo de dependencia emocional que caracteriza a los participantes de este estudio, se realizaron análisis estadísticos descriptivos con medidas de tendencia central, de dispersión y análisis de frecuencias. Posteriormente con base en el objetivo de determinar si existe una correlación entre autoestima de los participantes con la dependencia emocional, se realizó un análisis de correlación Rho de Spearman. Así entonces, en este apartado, se presentan los resultados de ambos análisis, así como los datos sociodemográficos de los mismos.

\section{Resultados descriptivos}

De acuerdo con los datos sociodemográficos de los participantes participaron 68 mujeres y 14 hombres y el promedio de edad fue de $M=21.52$ años ( $D E=1.92$ ), también indican que el tiempo de noviazgo promedio fue de $M=26.60$ meses ( $D E=24.46$ ), teniendo como tiempo mínimo de duración en el noviazgo 4 meses y como 
tiempo máximo 111 meses solo en un caso lo que indica que al momento del estudio la pareja llevaba 9 años 4 meses.

Por otro lado en cuanto al nivel de autoestima, se encontró que los participantes obtuvieron un nivel de autoestima baja $M=24$ ( $D E=5.06)$, por lo que presentan problemas significativos de autoestima, de acuerdo con los puntajes de las normas de calificación de la escala.

Respecto del nivel de dependencia emocional que presentaron los participantes, se puede observar que el promedio se ubicó en el percentil 19, lo cual representa al $19 \%$ del total de la muestra, siendo un $81 \%$ quienes se encuentran por arriba de este valor promedio. Es decir, estos participantes presentaron dependencia emocional en los rasgos contemplados dentro de ella.

Así mismo, se encontró que todos los rasgo de dependencia emocional, se presentan en este grupo de participantes, siendo la más frecuente la dependencia dominante.

Resultados de la correlación entre dependencia emocional y autoestima.

En la relación a los resultados de la correlación de los rasgos de dependencia emocional con autoestima, todas resultaron estadísticamente significativas a un nivel de significancia de .01 y de .05, como se puede apreciar en la siguiente tabla 1.

Tabla 1

Resultados de la Correlación Rho de Spearman entre rasgos de dependencia emocional y autoestima

\begin{tabular}{ll}
\hline Rasgo de dependencia emocional & Autoestima \\
\hline Necesidad excesiva del otro & -0.210 \\
Deseo de exclusividad en la relación & $-0.380^{* *}$ \\
Prioridad de la pareja sobre cualquier otra & -0.164 \\
cosa & \\
Idealización del objeto & $-0.224^{*}$ \\
Relaciones basadas en la sumisión y & $-0.223^{* *}$ \\
subordinación & \\
Historia de relaciones de pareja & $-0.448^{* *}$ \\
desequilibradas & \\
Miedo a la ruptura & $-0.350^{* *}$ \\
Características de los objetos de los & $-0.398^{* *}$ \\
dependientes emocionales & \\
Dependencia dominante & $-0.364^{* *}$ \\
Total de dependencia emocional & $-0.393^{* *}$ \\
\hline
\end{tabular}

Nota: $\mathrm{p} \leq .05^{*}, \mathrm{p} \leq .01 * *$

Los resultados de la correlación entre autoestima y la dimensión de necesidad excesiva del otro, no fue estadísticamente significativa $r_{s}=-0.210, \mathrm{p}=0.058$. De la correlación, entre autoestima y la dimensión de deseo de exclusividad en la relación, indica una fuerza de la relación media con $r_{s}=-0.380$, es inversamente proporcional, con una $p \leq 0.01$. Lo cual quiere decir que a menor nivel de autoestima, el deseo de exclusividad en la relación se incrementa.

La correlación entre autoestima y la dimensión de la priorización de la pareja sobre cualquier otra cosa, no fue estadísticamente significativa $r_{s}=-0.164, \mathrm{p}=0.139$. En cuanto a la correlación entre autoestima y la dimensión de la idealización del objeto, indica una fuerza de la relación baja con $r_{s}=-0.224$, es inversamente proporcional, con una $p \leq 0.05$. Lo cual quiere decir que a menor nivel de autoestima, la idealización del objeto se incrementa.

Respecto de la correlación entre autoestima y la dimensión de relaciones basadas en la sumisión y subordinación, indica una fuerza de la relación baja con $r_{s}=-0.223$, es inversamente proporcional, con una $\mathrm{p} \leq 0.01$. Lo cual quiere decir que a menor nivel de autoestima, las relaciones basadas en la sumisión y subordinación se incrementan.

Los resultados de la correlación entre autoestima y la dimensión de historia de relaciones de pareja desequilibradas, indica una fuerza de la relación media con $r_{s}=-0.448$, es inversamente proporcional, con una $\mathrm{p} \leq$ 0.01 . Lo cual quiere decir que a menor nivel de autoestima, aumentan las historias de relaciones de pareja desequilibradas.

Así mismo en la correlación entre autoestima y la dimensión de miedo a la ruptura, indica una fuerza de la relación media con $r_{s}=-0.350$, es inversamente proporcional, con una $p \leq 0.01$. Lo cual quiere decir que a menor nivel de autoestima, aumenta el miedo a la ruptura en la relación.

En cuanto a los resultados de la correlación entre autoestima y la dimensión de las características de los objetos de los dependientes emocionales, indica una fuerza de la relación media con $r_{s}=-0.398$, es inversamente proporcional, con una $p \leq 0.01$ Lo cual quiere decir que a menor nivel de autoestima, las características de los objetos de los dependientes emocionales se maximizan.

Los resultados de la correlación entre autoestima y la dimensión de dependencia dominante, indica una fuerza de la relación media con $r_{s}=-0.364$, es inversamente proporcional, con una $p \leq 0.01$. Lo cual quiere decir que a menor nivel de autoestima, la dependencia dominante, aumenta.

Por último, los resultados de la correlación entre autoestima y el puntaje total de dependencia emocional, indica una fuerza de la relación media con $r_{s}=-0.393$, es inversamente proporcional, con una $p \leq 0.01$. Lo cual quiere decir que a menor nivel de autoestima, aumenta la dependencia emocional. 


\section{Discusión}

De acuerdo con los resultados obtenidos, se puede apreciar que los estudiantes participantes en este estudio presentaron un nivel de autoestima que podría estimarse como baja, así como rasgos de conductas dependientes probablemente derivados de su propia percepción y valor personal disminuido.

Así mismo, existe una relación estadísticamente significativa entre autoestima y siete de las nueve dimensiones de dependencia emocional, por lo que se acepta la hipótesis planteada. De esas siete, cinco son correlaciones medias y dos son bajas, todas son inversamente proporcionales lo cual significa que a menor autoestima mayor dependencia emocional por parte de los participantes. Es de observar que la autoestima, no correlacionó con las dimensiones de dependencia emocional: necesidad excesiva del otro y priorización de la pareja sobre cualquier otra cosa.

Los hallazgos encontrados en este estudio, parecen ser coincidentes con los reportados por Narvaez (18), quien realizó un estudio para hallar la relación entre la dependencia emocional y la autoestima en estudiantes de un Centro Preuniversitario de Lima Norte en Perú, donde el $26.2 \%$ de los estudiantes reportó dependencia emocional. De igual forma, se encontró una relación positiva entre la dependencia emocional y autoestima.

Respecto de la baja autoestima presentada por los participantes de este estudio, como refieren Rodríguez, Pellicer y Domínguez (20) las personas con estas características suelen ser demasiado sensibles a las críticas por parte de los demás, sus pensamientos pueden llegar a ser negativos la mayor parte del tiempo, son más susceptibles a tener relaciones dependientes y a someterse a conductas de explotación por parte de los demás, motivo por el cual, la necesidad excesiva del otro se vio incrementada, así como la dependencia dominante y el miedo a la ruptura en la relación, aumenta.

Así entonces, como refieren Villa de la, Sirvent, Ovejero y Cuetos (21), las personas dependientes emocionalmente pasan por sentimientos negativos de soledad, tristeza, abatimiento, desánimo, culpa, insatisfacción, miedo a la soledad, deseos de autodestrucción. Muestran tendencia a acomodarse en las relaciones dejando que sean otros los que decidan por ellos y desarrollen una escasa o nula conciencias de problema.

Como citan Valle y Moral (22), la población joven puede llegar a presentar un mayor grado de dependencia emocional, como efecto de la prolongación de la adolescencia en sociedades en crisis, donde se ha dado lugar a un descenso en la competencia emocional y se valora la entrega incondicional y el sufrimiento como muestra de gran amor (23).

Por su parte, Mallma (24), quien analizó la relación entre relaciones intrafamiliares y dependencia emocional en estudiantes de licenciatura en psicología de la Universidad Autónoma de Lima. Perú, encontró en cuanto a la dependencia emocional que de los 467 participantes, el $64 \%$ de la muestra presentaban un nivel promedio en el factor de miedo a la soledad o abandono, seguido de un $28.1 \%$ (131) de la muestra que presenta un nivel Alto; también encontró que el $52 \%$ tuvo un nivel promedio en el factor búsqueda de aceptación y atención, seguida del $37.5 \%$ que presentaban un nivel alto. Además reportó que el $42.2 \%$ de los participantes, presentaron un nivel alto en el factor de apego y seguridad; un nivel promedio en idealización de la pareja; así como un puntaje promedio en el factor de percepción de su autoestima (como una de las áreas que se midieron de dependencia emocional), y el $50.1 \%$ se encontraba dentro de un nivel promedio en el puntaje total de dependencia emocional y sólo un $31.4 \%$ de la muestra puntúo en un dependiente emocional alto y muy alto.

Como se puede apreciar, un porcentaje de los estudiantes de psicología de la universidad de Perú, también llegan a presentar dependencia emocional, como en el caso de los estudiantes de esta institución universitaria donde se realizó el estudio.

$\mathrm{Si}$ bien se encontraron estos resultados, es importante destacar que hay otros estudios donde los participantes han obtenido puntajes altos en autoestima y las correlaciones de ésta con la dependencia emocional, también son inversamente proporcionales, como el realizado por Marín-Ocmin (13), con respecto a la relación entre dependencia emocional y autoestima, donde encontró que los estudiantes que presentan una autoestima alta no reportan dependencia emocional. Es probable que los hallazgos encontrados, se deban como señala Llanes y Leal (14), a la seguridad que la pareja le otorga a la persona dependiente, sin embargo, se considera pertinente seguir investigando sobre el tema a fin de confirmar esta posibilidad; así como también, contemplar los efectos que la dependencia emocional tienen en el rendimiento de los estudiantes universitarios, como se ha reportado por algunos autores.

También se sugiere seguir investigando respecto de la relación entre autoestima baja y las dimensiones de dependencia emocional: la necesidad excesiva del otro y priorización de la pareja sobre cualquier otra cosa, ya que en este caso no resultaron estadísticamente significativas. Tal vez se podría considerar otras variables como violencia en la pareja, en donde sí se han reportado relaciones entre autoestima baja y estas dos dimensiones, particularmente en estudios con mujeres, como lo reporta en su estudio Anaya (26). 
Con respecto a los análisis realizados en este estudio, cabe mencionar que no fue posible efectuar una comparación por sexo, debido al número de participante, por lo que se recomienda considerar una muestra más grande y equivalente en posteriores investigaciones.

De igual forma, resulta conveniente mencionar que los resultados de este estudio, deben ser considerados con cautela, dado que los instrumentos utilizados, si bien muestran una validez para población mexicana, en el caso de la escala de autoestima de Rosenberg, Jurado D., Jurado C., López, Querevalú (20), sugieren realizar más estudio para corroborar las propiedades psicométricas de su estudio.

\section{Conclusión}

Un aspecto relevante de este estudio, es que muestra que las personas baja autoestima pueden llegar a presentar algún rasgo de dependencia emocional, por lo que se pude decir, que si bien la autoestima puede ser un factor determinante, podría estar involucrados otros factores como son los rasgos de personalidad, la concepción de la relación de pareja, el nivel de resiliencia, los estilos de crianza, entre otras, las cuales se recomienda tener presente en futuras investigaciones.

Así mismo, resulta relevante considerar que los participantes de este estudio se encuentran en formación de la licenciatura en psicología, por lo que es importante prestar atención a programas que contribuyan al fortalecimiento de la autoestima, al considerar que al término de sus estudios universitarios, serán promotores de la salud mental. También se propone como conveniente abordar una perspectiva cualitativa para poder conocer a profundidad las experiencias de las personas que viven con dependencia emocional y observar los posibles efectos que ésta puede llegar a tener en su vida familiar, social y escolar.

Es de considerar que dadas las características de los instrumentos, baja validez del instrumento de dependencia emocional y la sugerencia de los autores que validaron la escala de Rosenberg en México de seguir investigando sobre las propiedades psicométricas, se sugiere en futuros estudios utilizar otros instrumentos que permitan verificar la relación entre ambas variables.

\section{Referencias}

[1] Lemos M, Londoño NH. Construcción y validación del Cuestionario de Dependencia Emocional en población colombiana. Acta Colombiana de Psicología 2006; 9: 127-140.

[2] Morales M. Dependencia emocional: mujeres que no saben amar por baja autoestima. [Tesina profesional] Universidad Nacional Autónoma

$$
\begin{array}{lccc}
\text { de } & \text { México; } & 2019 . & \text { Disponible } \\
\text { http://132.248.9.195/ptd2019/julio/0792407/Index.html }
\end{array}
$$

[3] Sirvent C, Moral MV. Construcción y validación del Inventario de Relaciones Interpersonales y Dependencias Sentimentales (IRIDS100). Health and Addictions 2018; 18(2): 35-47.

[4] Santamaría JJ, Merino L, Montero E, Cano M, Fernández T, Cubero P, López O, González-Bueso V. Perfil psicopatológico de pacientes con dependencia emocional. Revista Iberoamericana de Psicosomática 2015; 116: 36-46. Disponible en: file://C:/Users/NORMA/Downloads/Dialnet-

PerfilPsicopatologicoDePacientesConDependenciaEmoc-5288486.pdf

[5] Castelló J. Dependencia emocional características y tratamiento. Madrid: Alianza; 2005.

[6] Moral MV, Sirvent C. Dependencias sentimentales o afectivas: etiología, clasificación y evaluación. Revista Española de Drogodependencias 2008; 33: 145-167

[7] Asociación Americana de Psiquiatría. Manual Diagnóstico de los Trastornos Mentales (4ta ed.). Masson; 2002.

[8] American Psychiatric Association Manual Diagnóstico de los Trastornos Mentales (5ta ed.). 2013. Disponible en: htttps//doi.org/1176/appi.books.9780890425596

[9] Rodríguez-Franco L, López-Cepero J, Rodríguez-Díaz FJ, AntuñaBellerín MA. La violencia de género entre parejas de novios universitarios. En: I. Vázquez, Coord. Libro de Actas del I Congreso Universitario Andaluz "Investigación y Género" Sevilla: Universidad de Sevilla; 2009: 1123-1143.

[10] Pradas E, Perles F. Resolución de conflictos de pareja en adolescentes, sexismo y dependencia emocional. Quaderns de Psicología 2012; 14 (1): $45-60$

[11] Urbiola I, Estévez A, Iruarrizaga I, Jauregui P. Dependencia emocional en jóvenes: relación con la sintomatología ansiosa y depresiva, autoestima y diferencias de género. Ansiedad y Estrés 2017; 23: 6-11. Disponible en: https://doi.org/10.1016/j.anyes.2016.11.003

[12] De la Villa M, García A, Cuetos G, Sirvent C. Violencia en el noviazgo, dependencia emocional y autoestima en adolescentes y jóvenes españoles. Revista Iberoamericana de Psicología y Salud 2017; 8(2): 96-107. Disponible en: https://doi.org/10.23923/j.rips.2017.08.009

[13] Marín-Ocmin A. Dependencia emocional y autoestima: relación y características en una población de jóvenes limeños. CASUS 2019; 4(2): 85-91. Disponible en: file://C:/Users/NORMA/Downloads/Dialnet-

DependenciaEmocionalYAutoestima-7083955\%20(1).pdf

[14] LLanes H, Leal S. Revisión documental sobre dependencia emocional en las relaciones de pareja. Universidad Cooperativa de Colombia, Facultad de Psicología Santa Marta; 2017. Disponible en: https://repository.ucc.edu.co/bitstream/20.500.12494/11036/1/2017_d ependencia_emocional_pareja.pdf

[15] Hertfelder C. Poder ser Yo. La autoestima en la familia. Ediciones Palabra; 2019. Disponible en: https://books.google.com.mx/books?id=hIK6DwAAQBAJ\&pg=PT11 6\&lpg=PT116\&dq=relaci\%C3\%B2n+entre+autoestima+y+apego+ins eguro\&source $=$ bl\&ots $=$ hEplXbTy $1 \&$ sig $=$ ACfU3U2AU8UsW5cHex hrk_E1ypuokGmbQ\&hl=es\&sa $=X \& v e d=2 a h U K E w j U t N 36 s 4 X r A h U F W a 0 K H$ ezdAUU4lgEQ6AEwEnoECAoQAQ\#v=onepage \&q=relaci\%C3\%B2 $\mathrm{n} \% 20$ entre\%20autoestima\%20y\%20apego\%20inseguro\&f=false 
[16] Lozano-Sapuy L.P., Albarracín-Ángeles P.A., Vázquez-Amézquita M. Apego parental y su relación con el apego romántico y la dependencia afectiva en 119 universitarios de la ciudad de Ibagué-Colombia Revista de Psicología GEPU 2016; 7 (1): 8-39. Disponible en: https://revistadepsicologiagepu.es.tl/Apego-Parental-y-su-relaci\%F3ncon-el-Apego-Rom\%E1ntico-y-la-Dependencia-Afectiva-en-119-

Universitarios-de-la-ciudad-de-Ibagu-e2--\%26\%238211\%3B-

Colombia.htm

[17] Cajo E. Dependencia emocional y conflictos en la relación de pareja, en estudiantes de un instituto - Jaén [tesis de pregrado]. Universidad Señor de Sipán, Pimentel - Perú; 2013.

[18] Guerra R, Mego C. Dependencia emocional y violencia en las relaciones de pareja en estudiantes de la escuela de Derecho de la Universidad Señor de Sipán [tesis de pregrado]. Universidad Señor de Sipán, Pimentel - Perú; 2011.

[19] Narvaez MO. Relación entre la dependencia emocional y la autoestima en jóvenes universitarios de la carrera profesional de enfermería de la Universidad Católica Sedes Sapientiae [Tesis de licenciatura]. Universidad Católica Sedes Sapientiae, Lima Perú; 2018. Disponible en:

http://repositorio.ucss.edu.pe/bitstream/handle/UCSS/575/Narvaez_M arleny_tesis_bachiller_2018.pdf?sequence $=1$ \&isAllowed=y

[20] Jurado D, Jurado C, López k; Querevalú B. Validez de la Escala de Autoestima de Rosenberg en universitarios de la Ciudad de México. Revista Latinoamericana de Medicina Conductual 2015; 5(1): 18-22.

[21] Rodríguez M., Pellicer G., Domínguez M. Autoestima: clave del éxito personal. México, D.F.: Manual Moderno; 2012.

[22] Villa de la Ma, Sirvent, C, Ovejero, A, Cuetos, G. Dependencia emocional en las relaciones de pareja como Síndrome de Artemisa: modelo explicativo. Terapia Psicología 2018; 36(3): 156-166. Disponible en: https://scielo.conicyt.cl/pdf/terpsicol/v36n3/0718 4808-terpsicol-36-03-0156.pdf

[23] Valle L, Moral MV. Dependencia emocional y estilo de apego adulto en las relaciones de noviazgo en jóvenes españoles. Revista Iberoamericana de Psicología y Salud 2018; 9(1): 27-41. Disponible en: http://dx.doi.org/10.23923/j. rips.2018.01.013

[24] Galán-Jiménez SF, Sánchez-Armáss-Cappello O. Psychometric properties of the dependent ideation scale. Anales de Psicología / Annals of Psychology 2018; 34(3): 465-471. Disponible en: https://doi.org/10.6018/ analesps.34.3.318471

[25] Mallma N. H. Relaciones intrafamiliares y dependencia emocional en estudiantes de psicología de un centro de formación superior de Lima Sur [Tesis de licenciatura]. Universidad Autónoma de Perú, Facultad de Humanidades, Lima Perú; 2014.

[26] Anaya CE. Dependencia emocional en mujeres víctimas y no víctimas de violencia psicológica de lima metropolitana [tesis de pregrado]. Universidad de San Ignacio de Loyola- Perú; 2020. Disponible en: http://repositorio.usil.edu.pe/bitstream/USIL/10270/1/2020_Anaya\%2 OVargas.pdf 